\title{
Thrombophilia with an onset symptom of intracranial venous thrombosis: A case report and review of the literature
}

\author{
LI CUI $^{1}$, LIJUN ZHU ${ }^{2}$, YUTING WANG ${ }^{1}$, WUQIONG ZHANG ${ }^{1}$ and SHAOKUAN FANG ${ }^{1}$ \\ ${ }^{1}$ Department of Neurology, Neuroscience Center, The First Hospital of Jilin University, Changchun, Jilin 130012; \\ ${ }^{2}$ Department of Neurology, The Third Teaching Hospital of Jilin University, Changchun, Jilin 130021, P.R. China
}

Received December 28, 2015; Accepted February 7, 2017

DOI: $10.3892 / \mathrm{etm} .2017 .4316$

\begin{abstract}
Thrombophilia may be hereditary or acquired and is associated with a high risk of thrombosis. The diagnosis rate for thrombophilia is low, particularly for patients with non-specific symptoms. The present study describes a patient with thrombophilia, presenting with onset symptoms for intracranial venous thrombosis. The patient had increased serum homocysteine and anticardiolipin immunoglobulin $\mathrm{G}$ antibodies and decreased protein $\mathrm{S}$ activities. In addition, the patient was obese and had a one-week history of fatigue, immobilization and insufficient water intake. Radiological findings identified multiple venous thrombosis. Since the patient had multiple risk factors for thrombosis, the diagnosis of thrombophilia was made. No mutations with definite clinical significance were identified in the assessments for mutations of the protein S- $\alpha$ (PROS1) gene. The current case highlights the importance of correct diagnosis for thrombophilia in patients who present with the onset symptoms of intracranial venous thrombosis.
\end{abstract}

\section{Introduction}

Thrombophilia is a hereditary or acquired tendency to a hypercoagulable state that increases the risk of venous thromboembolism (VTE) and primarily deep venous thrombosis (DVT) as a consequence of genetic and environmental risk factors, including a protein $\mathrm{S}-\alpha$ mutation in protein $\mathrm{S}$ and immobilization, respectively (1). Thrombophilia was initially identified in a patient with a hereditary antithrombin deficiency in 1965 (2). Hereditary thrombophilia may be caused primarily by deficiencies of natural anticoagulants including prothrombin, factor $\mathrm{V}$ Leiden, protein $\mathrm{C}$ and protein $\mathrm{S}$ with a prevalence of $<1 \%$ in the general population (3). A

Correspondence to: Professor Shaokuan Fang, Department of Neurology, Neuroscience Center, The First Hospital of Jilin University, 71 Xinmin Street, Changchun, Jilin 130012, P.R. China E-mail: fang20063536@sina.com

Key words: thrombophilia, venous thromboembolism, protein $\mathrm{S}$ alpha 1 gene, intracranial venous thrombosis number of conditions, such as antiphospholipid syndrome (APS) and malignant cancer are associated with acquired thrombophilia $(4,5)$. Anticoagulation is the primary treatment regardless of the cause (6), typically administered for 5 days followed by oral anticoagulants for 6 months. The most common condition associated with thrombophilia is venous thromboembolism (VTE), including superficial thrombosis and DVT, which in turn leads to mortality and pulmonary embolism, with a prevalence of 56-160 in 100,000 individuals per year, which represents a major cause of morbidity and mortality in thrombophilia (7).

Although intracerebral thrombosis induced by thrombophilia is not very rare it may be susceptible to misdiagnosis. The present study reports a case of a 24-year-old male with thrombophilia, who presented with onset symptoms of intracranial venous thrombosis.

\section{Case report}

A 24-year-old male was admitted to the Department of Neurology, the First Teaching Hospital of Jilin University (Changchun City, China) in June 2015 following a seizure and $2 \mathrm{~h}$ of unconsciousness. Prior to admission, the patient had fallen on his head while playing basketball and had seizure symptoms including clenched teeth, closed fists, rigid extremities, tongue biting and foaming at the mouth. A second seizure attack occurred 7 to 8 min later. The patient was unconscious during and between the seizure attacks and received an intravenous injection of diazepam, mannitol, glycerol fructose and piracetam. Following the intravenous administration the patient was restless. A week prior to admission, the patient had presented with fatigue, immobilization, and insufficient water intake. The patient was healthy with no previous history of seizure, hypertension, diabetes or cardiovascular disease. There was also no history of recurrent headache or unconsciousness prior to the fall. The patient had a history of smoking 20 cigarettes per day for 5 years and infrequent alcohol consumption.

On examination, the patient was unconscious with decreased pupillary light reflex. Body temperature was $38.7^{\circ} \mathrm{C}$ and blood pressure was $127 / 66 \mathrm{mmHg}$. Normal muscle tone and strength were observed. Babinski's sign was bilaterally positive, demonstrated by an over-extension and fanning of the toes following stimulation, while a negative result would 
A

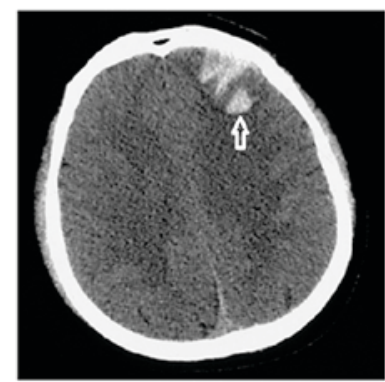

$\mathrm{B}$

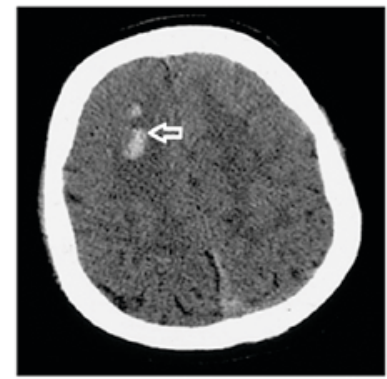

$\mathrm{C}$

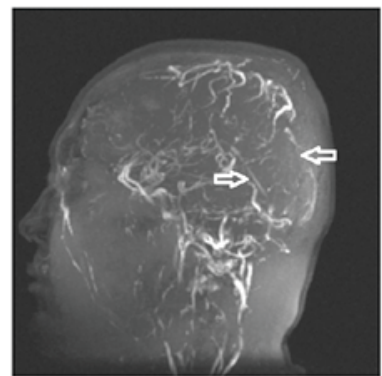

Figure 1. Computed tomography and magnetic resonance venography images of the patient on admission. Cranial computed tomography at admission demonstrated (A) intracerebral hemorrhage in the bilateral frontal lobe with (B) subarachnoid hemorrhage. (C) A cranial magnetic resonance venography image revealed the absence of signals in the sinus sagittalis superior and sinus rectus and thread-like signals in the transverse sinus. White arrows indicate (A) intracerebral hemorrhage, (B) subarachnoid hemorrhage and (C) absence of signals.

be a normal flexion of the toes. White blood cell count was $26.14 \times 10^{9} / 1$ (normal range: $3.5-9.5 \times 10^{9} / 1$ ) and neutrophils $14.79 \times 10^{9} / 1$ (normal range: $1.8-6.3 \times 10^{9} / 1$ ). A number of the values observed from the blood lipid profile and liver enzyme examinations, completed as routine administration on admission, were elevated, as follows: aspartate aminotransferase 190.5 U/1 (normal range: 13-35 U/1), alanine aminotransferase $587.7 \mathrm{U} / 1$ (normal range: 7-40 U/1), $\gamma$-glutamyl transpeptidase $115.4 \mathrm{U} / 1$ (normal range: 7-45 U/1), triglycerides $7.65 \mathrm{mmol} / \mathrm{l}$ (normal range: $0.28-1.80 \mathrm{mmol} / \mathrm{l}$ ), however, the high density lipoprotein $0.73 \mathrm{mmol} / 1$ (normal range: 0.76-2.10 mmol/l) indicated a normal value. A liver ultrasound revealed that the patient had a fatty liver with normal portal vein circulation Cranial computed tomography (CT) on admission indicated intracerebral hemorrhage in the bilateral frontal lobes with subarachnoid hemorrhage (Fig. 1A and B). Computerized tomography angiography (CTA) of the head revealed no abnormality. Cranial magnetic resonance imaging (MRI) demonstrated multiple abnormal signals in the bilateral frontal lobes. The patient was diagnosed with a brain hemorrhage with malacic and cystic lesions. Cranial magnetic resonance venography (MRV) was completed using Siemens Trio Tim 3.0T scanner (Siemens AG, Munich, Germany) revealed an absence of signal in the sinus sagittalis superior and sinus rectus and thread-like signals in the transverse sinus (Fig. 1C). Lumbar puncture revealed a cerebrospinal fluid (CSF) pressure of $350 \mathrm{~mm} \mathrm{H}_{2} \mathrm{O}$. Biochemical and cytological examination of the CSF had normal results, as follows: CSF protein $0.32 \mathrm{~g} / 1$ (normal range: $0.15-0.45 \mathrm{~g} / \mathrm{l}$ ); glucose $3.78 \mathrm{mmol} / 1$ (normal range: $2.3-4.1 \mathrm{mmol} / \mathrm{l}$ ); chloridion $128 \mathrm{mmol} / 1$ (normal range: 119-129 mmol/1); white blood cell: $3 \times 10^{6} / 1$ (normal range: 0-8x10\%/1); red blood cell: 0 (normal value: 0 ) and Pandy reaction: negative. Other examinations including immunological examination, thyroid function, rheumatic anti neutrophil cytoplasmic antibodies, erythrocyte sedimentation rate, rheumatoid factors, $\mathrm{C}$-reactive protein, and hormone concentrations were normal. The patient was diagnosed with cerebral venous sinus thrombosis.

During hospitalization, the patient complained of swelling and pain in the left leg. An ultrasound of the extremities demonstrated a bilateral cephalic venous thrombosis in the subacute phase and left posterior tibial venous thrombosis in the acute-subacute phase. Subcutaneous edema was identified in the left upper arm using a Color Doppler ultrasound. Color Doppler ultrasound of the neck revealed bilateral narrowing of

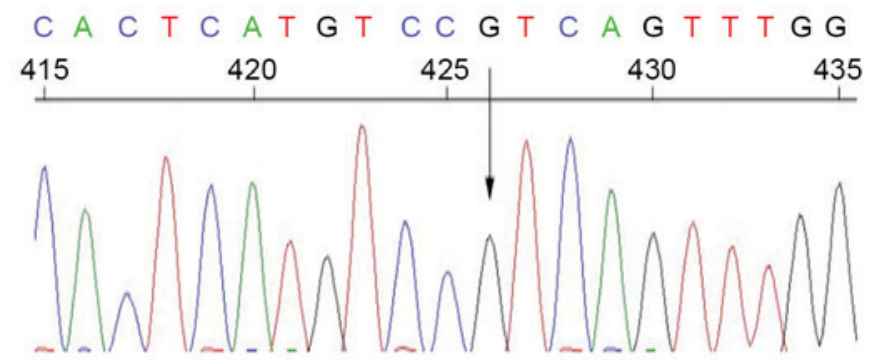

Figure 2. Gene map. The genetic sequence results of the protein S- $\alpha$ (PROS1) gene. The arrow indicates a single polymorphism variation (c. 2001A>G, p. Pro667Pro) in exon 5 of the PROS1 gene.

the jugular vein with the predominant narrowing of the right side and venous return disorder in the $\mathrm{J} 2$ and $\mathrm{J} 3$ segments of the right internal jugular vein. Laboratory tests of the blood revealed the following values: Anticardiolipin immunoglobulin Ig G antibody $14 \mathrm{U} / \mathrm{ml}$ (normal value: 0-10 U/ml), serum homocysteine $21.6 \mu \mathrm{mol} / 1$ (normal value: $0-15 \mu \mathrm{mol} / \mathrm{l}$ ), protein S $53.4 \%$ (normal value: $55-130 \%$ ), protein C $129 \%$ (normal value: $70-140 \%$ ), antithrombin $136 \%$ (normal value: $80-120 \%$ ), fibrinogen $124 \%$ (normal value: $80.2-132.5 \%$ ) and neuron-specific marker enolase $26.25 \mathrm{ng} / \mathrm{ml}$ (normal value: $<25.00 \mathrm{ng} / \mathrm{ml}$ ). The patient was diagnosed with thrombophilia and the parents of the patient were observed to have normal extremity ultrasound results.

As the patient presented with a protein $\mathrm{S}$ deficiency, examinations for mutations of the protein $\mathrm{S}-\alpha$ (PROS1) gene were completed. A single genetic change (c. 2001A>G, p. Pro667Pro) was identified in exon 5 of the PROS1 gene (Fig. 2). Based on the single nucleotide polymorphism database of SNP (http://www.ncbi.nlm.nih.gov/SNP/) this genetic change was a polymorphism variation, not a clinically relevant mutation.

The patient received dehydration therapy in combination with the use of a low dose of molecular weight heparin (5,000 IU once a day) and liver-protecting agent, Reduced glutathione (Beijing Zhongsheng Ruitai Biotechnology, Co., Ltd., Beijing, China) $400 \mathrm{mg}$ three times daily for 15 days. No seizure or headache occurred during 28 days of hospitalization. The hematoma was absorbed, as observed in cranial CT examinations and $\mathrm{CSF}$ pressure was $350 \mathrm{~mm} \mathrm{H}_{2} \mathrm{O}$. Venous ultrasound of the extremities demonstrated a subacute superficial thrombophlebitis in the bilateral distal cephalic vein and the forearm segment of the right basilic vein. The level of liver 
aminotransferase decreased to be within the normal range. The patient was discharged from the hospital and followed up once a month for 6 months. The patient received oral warfarin from initial dosage of $3 \mathrm{mg}$ up to $4.5 \mathrm{mg}$ following discharge. The patient had an international normalized ratio (INR) of 2.0 at the most recent follow-ups (December, 2015).

\section{Discussion}

Thrombophilia is an inherited or acquired condition that predisposes patients to thrombosis. The common causes of hereditary thrombophilia are deficiency of coagulation inhibitors including protein $\mathrm{C}$, protein $\mathrm{S}$ and antithrombin and genetic mutations such as FV Leiden, prothrombin G20210A mutation and a MTHF R677T mutation (2). Acquired thrombophilia is commonly associated with diseases including APS (8), paroxysmal nocturnal hemoglobinuria (9), myeloproliferative disorders (9) and malignant cancer (10), in addition to prolonged immobilization, trauma and necessitating surgery (5). The most common cause of acquired thrombophilia is APS, which is caused by the lupus anticoagulant and anticardiolipin antibody (8). In the present case, the patient had increased levels of serum homocysteine and anticardiolipin IgG antibodies and decreased protein $\mathrm{S}$ activities. In addition, the patient was obese and had a one-week history of fatigue, immobilization and insufficient water intake. Radiological findings indicated multiple venous thrombosis. Since the patient had multiple risk factors for thrombosis, the diagnosis of thrombophilia was made. Tests for the PROS1 gene were negative, although a polymorphism variation in the PROS1 gene was identified. Acquired thrombophilia was considered to be the predominant cause of thrombosis for this patient. Additionally, the patient had a slightly increased level of enolase, a tumor-specific marker, which was not clinically significant. The patient was asked to complete a follow-up examination, after 1 month, of the enolase concentration for the differential diagnosis of malignant cancer and the value was observed to return to normal ( $20.55 \mathrm{ng} / \mathrm{ml}$; normal range $<25 \mathrm{ng} / \mathrm{ml})$.

The patient had an elevated aminotransferase level during hospitalization. Although ultrasound revealed no abnormality in the portal vein, the potential for the liver function to be impaired due to portal vein thrombosis requires consideration. Since the aminotransferase level decreased following liver-protecting therapy, CT was not performed during hospitalization to examine whether portal vein thrombosis existed. If the aminotransferase is elevated during follow-up, CT should be performed to identify whether portal vein thrombosis is present or not.

There are a number of important coagulation inhibitors that keep the balance between fibrinolysis and thrombosis. Thromboembolic events occur as a result of the disruption of the balance leading to a hypercoagulable state (11). Coagulation inhibitor deficiency is present in $\sim 10 \%$ of the Caucasian population with deep vein thrombosis, and antithrombin deficiency is the predominant cause (12). By contrast, in the Asian population with deep vein thrombosis including Chinese, Japanese and Korean individuals, coagulation inhibitor deficiency is identified in $\sim 50 \%$ of the population, and the predominant cause is protein $\mathrm{S}$ deficiency (13). Suehisa et al (14) demonstrated that protein S deficiency was present in 20/32 Japanese patients with deep vein thrombosis. TFPI polymorphisms are associated with VTE, but it remains to be determined whether these polymorphisms are risk factors for VTE (14). The most common form of hereditary hemophilia is FV Leiden, a mutation in the factor $\mathrm{V}$ gene resistant to cleavage by activated protein C (APC) (15). FV Leiden may lead to a hypercoagulable state as the result of less anticoagulant activity of APC. The frequency of FV Leiden mutations varies among different ethnicities and regions, and the incidence is highest in the Caucasian population with $2-15 \%$ (16). Similarly, the prothrombin G20210A mutation is most frequently identified in the Caucasian population (12), but is rare amongst the Asian population (13). In addition, homozygous methylenetetrahydrofolate reductase (MRHFR) 677TT mutation is associated with increased blood homocysteine levels, and is a common cause of hyperhomocysteinemia (11). Although the MRHFR TT genotype is associated with mild hyperhomocysteinemia, which is regarded as a moderate risk for venous thromboembolism (13), a previous study has indicated that an increase in the blood homocysteine levels is only a mild risk factor for venous thrombosis (17).

It remains unclear whether all individuals with thrombosis should be screened for thrombophilia. Screening is generally not recommended for the general population. However, a number of specific indications are recommended for screening initiated by the Anticoagulation Forum (9), these include i) idiopathic or recurrent venous thromboembolism, ii) a family history of venous thromboembolism, specifically first-degree relatives with an early onset of venous thromboembolism, iii) recurrent miscarriage $(>3)$, iv) thrombosis during perinatal period or following the use of oral contraceptives or estrogen replacement therapy (9), v) neonates with visceral thrombosis, fulminant purpura and hemorrhagic skin necrosis, vi) venous thromboembolism in uncommon sites such as the brain, liver, mesentery or renal vein, vii) younger patients ( $<40$ years) with early onset venous thromboembolisms and viii) thrombosis or skin necrosis during warfarin therapy. However, for patients with malignant cancer, screening for thrombophilia is generally not recommended, because such patients receive long-term anticoagulation therapy (18).

Currently, prophylactic anticoagulant therapy is recommended for patients with a high risk of thromboembolism, and it is recommended that the INR be controlled between 2 and $3(19,20)$. These high-risk patients include: i) Patients with recurrent thromboembolism ( $>2$ occurrences), ii) patients with antithrombin deficiency or APS who have experienced at least one thromboembolic event, iii) patients who experience severe thrombosis such as severe pre-eclampsia, cerebrovascular thrombosis, mesenteric artery thrombosis or portal vein thrombosis, requiring lifelong direct oral warfarin and higher INR (between 2.5-3.0), iv) patients who had thrombosis at an unusual site such as mesenteric vein or cerebral vein and v) patients with $>1$ risk factor for thrombophilia who experience at least one thromboembolic event. For patients with first onset of acute thromboembolism, regular heparin or low-molecular heparin should be administered for at least 5 days, followed by oral anticoagulants for 6 months, with the exception of pregnant women, due to the potential of anticoagulants to cause pregnancy loss. The INR should be controlled between 2 and 3, and may be higher for recurrent 
thrombosis during anticoagulation therapy. With the exception of the case of acute pulmonary embolism, thrombolytic therapy is not required (21). In the present case, since the patient had subdural hematoma, only low molecular heparin was administered for 3 weeks during hospitalization and oral warfarin following discharge. The patient had an INR of 2.0 at the most recent follow-ups in December 2016.

The clinical diagnosis rate for thrombophilia is low (3). A number of reasons may cause this low diagnosis rate. Firstly, the symptoms are unspecific, particularly in cases with small thrombi. Certain patients may only present with palpitations, chest tightness or transient dizziness and thus, may be misdiagnosed. Secondly, it is easy to misdiagnose thrombophilia as intracranial venous thrombosis, phlebitis, pulmonary embolism, acute myocardial infarction, volvulus and necrotic enteritis. Tests for thrombophilia are often neglected following the initial diagnosis of these diseases. Thirdly, due to the incidence of thrombophilia being low, doctors often lack sufficient awareness of this disease. Finally, the diagnosis of thrombophilia is dependent on a number of laboratory tests, including antithrombin, clotting factors and genetic mutations of numerous associated genes, some of which are difficult to perform due to interference factors such as age, gender and the use of oral contraceptives (6). In the present case, the diagnosis of thrombophilia was made based on laboratory testing of antithrombin, APS antibodies, serum homocysteine levels, protein $\mathrm{C}$, protein $\mathrm{S}$ and fibrinogen in addition to ultrasonic examination of the extremities and MR, CT and CTA examination of the brain. In addition, a number of predisposing factors for thrombosis were excluded such as surgery, trauma, fracture, malignant tumor and immobility due to chronic diseases.

For a patient $<60$ years old with recurrent venous thrombosis, thrombophilia should be considered for diagnosis (1). Early diagnosis of thrombophilia may prevent the occurrence of severe life-threatening diseases, such as pulmonary thrombosis. The current study presented a rare case of thrombophilia with an onset of intracerebral venous thrombosis. The present study also demonstrated that genetic testing is an alternative approach to define the risk of VTE, which is a limitation of screening test. Further studies are required to reveal the mechanism and provide an update on diagnosis, treatment options and prognosis.

\section{References}

1. Vilhena J, Vicente H, Martins MR, Grañeda JM, Caldeira F, Gusmão R, Neves J and Neves J: A case-based reasoning view of thrombophilia risk. J Biomed Inform 62: 265-275, 2016.

2. Egeberg O: On the natural blood coagulation inhibitor system. Investigations of inhibitor factors based on antithrombin deficient blood. Thromb Diath Haemorrh 14: 473-489, 1965.
3. Cohn DM, Vansenne F, de Borgie CA and Middeldorp S: Thrombophilia testing for prevention of recurrent venous thromboembolism. Cochrane Database Syst Rev 12: CD007069, 2012.

4. Falanga A: Thrombophilia in cancer. Semin Thromb Hemost 31: 104-110, 2005.

5. Armstrong EM, Bellone JM, Hornsby LB, Treadway S and Phillippe HM: Acquired Thrombophilia. J Pharm Pract 27: 234-242, 2014

6. Korovin LN, Raoof M, Kettelle JB, McClenathan JH and Patel JA: Cocurrent factor $\mathrm{V}$ Leiden and protein $\mathrm{C}$ deficiency presenting as mesenteric venous thrombosis. Am Surg 82: E96-E98, 2016.

7. East AT and Wakefield TW: What is the optimal duration of treatment for DVT? An update on evidence-based medicine of treatment for DVT. Semin Vasc Surg 23: 182-191, 2010.

8. Khamashta M, Taraborelli M, Sciascia S and Tincani A: Antiphospholipid syndrome. Best Pract Res Clin Rheumatol 30: 133-148, 2016.

9. Stevens SM, Woller SC, Bauer KA, Kasthuri R, Cushman M, Streiff M, Lim W and Douketis JD: Guidance for the evaluation and treatment of hereditary and acquired thrombophilia. J Thromb Trombolysis 41: 154-164, 2016.

10. Ay C, Pabinger I and Cohen AT. Cancer-associated venous thromboembolism: Burden, mechanisms and management. Thromb Haemost 117: 219-230, 2017.

11. Ekim M, Ekim H and Y1lmaz YK: The prevalence of Factor V Leiden, prothrombin G20210A, MTHFR C677T and MTHFR A1298C mutations in healthy Turkish population. Hippokratia 19: 309-313, 2015.

12. Philipp CS, Faiz AS, Beckman MG, Grant A, Bockenstedt PL, Heit JA, James AH, Kulkarni R, Manco-Johnson MJ, Moll S and Ortel TL: Differences in thrombotic risk factors in black and white women with adverse pregnancy outcome. Thromb Res 133: 108-111, 2014.

13. Tang L, Jian XR, Hamasaki N, Guo T, Wang HF, Lu X, Wang QY and $\mathrm{Hu} \mathrm{Y}$ : Molecular basis of protein S deficiency in China. Am J Hematol 88: 899-905, 2013.

14. Suehisa E, Nomura T, Kawasaki T and Kanakura Y: Frequency of natural coagulation inhibitor (antithrombin III, protein C and protein $S$ ) deficiencies in Japanese patients with spontaneous deep vein thrombosis. Blood Coagul Fibrinolysis 12: 95-99, 2001.

15. Cinier G, Öz A, Tekkesin AI, Hayıroğlu Mİ, Keskin M and Avsar Ş: A young male patient with multiple thromboembolism associated with factor V Leiden mutation. Int Heart J 57: 654-656, 2016.

16. Boroumand M, Pourgholi L, Ziaee S, Anvari MS, Jalali A and Goodarzynejad $\mathrm{H}$ : The association between factor V Leiden with the presence and severity of coronary artery disease. Clin Biochem 47: 356-360, 2014.

17. Lockwood C and Wendel G; Committee on Practice BulletinsObstetrics: Practice bulletin no. 124: inherited thrombophilias in pregnancy. Obstet Gynecol 118: 730-740, 2011.

18. Cunningham MT, Olson JD, Chandler WL, Van Cott EM, Eby CS, Teruya J, Hollensead SC, Adcock DM, Allison PM, Kottke-Marchant KK and Smith MD: External quality assurance of antithrombin, protein $\mathrm{C}$, and protein s assays: results of the College of American Pathologists proficiency testing program in thrombophilia. Arch Pathol Lab Med 135: 227-232, 2011.

19. James AH: Thromboembolism in pregnancy: Recurrence risks, prevention and management. Curr Opin Obstet Gynecol 20: 550-556, 2008

20. Bauer KA: Management of thrombophilia. J Thromb Haemost 1: 1429-1434, 2003.

21. Favaloro EJ: Diagnostic issues in thrombophilia: A laboratory scientist's view. Semin Thromb Hemost 31: 11-16, 2005. 